\title{
MOLECULAR TARGETS FOR LOW LEVEL LIGHT THERAPY
}

\author{
Kendric C. Smith \\ Stanford University School of Medicine, Stanford, CA, USA
}

\begin{abstract}
There are many molecular targets for Low Level Light Therapy (LLLT). The chief target appears to be cytochrome $\mathrm{C}$ oxidase in mitochondria, which then initiates a cascade of molecular events that signal the cell to perform various functions. Although visible light can produce photochemistry, infrared radiation only produces molecular rotations and vibrations. A probable explanation of how $633 \mathrm{~nm}$ radiation can produce the same biological effect as $904 \mathrm{~nm}$ radiation will be presented. The light activation of enzymes by multiple mechanisms will be discussed. To further enhance the scientific basis of LLLT, I propose that gene array experiments be performed at the wavelengths that have been shown to be most effective for different therapies with LLLT. Then we will know what genes are activated by the different wavelengths, and therapy can then be designed with greater confidence for success. It will also help us to determine if the use of more than one wavelength in a therapy might be advantageous. Think of the different wavelengths of light as different drugs, therefore it is important to determine which drug is best, and also the optimum dose.
\end{abstract}

In a recent article, ${ }^{1)}$ I discussed the basics of photobiology; what light does and does not do, and the fact that lasers are just expensive flashlights, and it is the light produced by the laser that produces the biological effect. Lasers are not magical, they are just a source of light.

I also pointed out that the major reason that LLLT has been slow to achieve the reputation that it deserves in the medical and scientific communities is because so many bad papers have been published by people who are not trained in science methodology, and who know nothing about photobiology. These bad papers are then accepted for publication by reviewers and editors who themselves are not trained in science or photobiology. I gave several examples.

Not only are bad papers published in journals, but bad books are also published that do more harm than good for LLLT. This includes one book ${ }^{2)}$ that contains many

Addressee for Correspondence:

Kendric C. Smith, Ph.D.

927 Mears Court, Stanford, CA 94305

E-mail: kendric@stanford.edu

Website: http://www.stanford.edu/ kendric/ errors, including crediting me as the author of the First Law of Photochemistry. I am old, but not that old. Actually, it is the Grotthus Draper Law from the early 1880's.

Now, I want to discuss the GOOD science in LLLT, and possible NEW approaches to understanding the targets for phototherapy. Please note that I used the word phototherapy, since that is what LLLT is.

The science of photobiology can be divided into 13 major specialty areas. Of these, four are relevant to LLLT, because they require the reception of only a few photons of light, and then transfer the information in this light to other molecules in the cell, which ultimately perform the required action.

1. Non-Visual Photoreception. Light is received by a receptor in an organism to monitor the environment without forming an image, in contrast to the case for Vision. A few examples are the circadian clock, which controls hormonal levels in birds and animals, and photoperiodism, which controls seasonal growth in

Manuscript received: August 16th, 2010

Accepted for publication: September 8th, 2010

Laser Therapy 19.3: 135-142 
plants and animals.

The receptor for controlling circadian rhythms in humans is melanopsin, a non-visual photoreceptor, which resides in the eyes, and absorbs blue light. For plants, the photoreceptors are cryptochromes and phytochromes.

2. Vision. This photoreception results in the formation of an image. This field covers the structure and photochemistry of the visual pigments in the rod and cone photoreceptors of eyes. The first step in vision is the photoisomerization of 11 -cis retinal to form all-trans retinal.

3. Photomorphogenesis. The development of an organism can be influenced by the information in light. This information comes from the quantity, the quality (i.e., the wavelengths present), the direction from which the light comes, and the periodicity of the light. There are blue light, red light, and far-red light sensors. The blue light receptors, cryptochrome and phototropin, act as sensors for the quantity of light, and respond accordingly.

The photoreceptor molecule, phytochrome, is reversibly inter-convertible between its inactive $\mathrm{Pr}$ form, and its active Pfr form by the sequential absorption of red and far-red light. Pfr formation, i.e., signal perception, initiates a transduction process that culminates in the altered expression of selected genes, and ultimately in altered growth and development appropriate for the prevailing light environment.

4. Photomovement. To produce movement, plants and organisms depend upon the quality and the direction of the light striking their photoreceptors. In photokinesis, an organism swims toward or away from light. Phototropic curvature in plants can occur toward or away from the light.

You can find chapters on all aspects of photobiology, including several chapters on LLLT on the website for Photobiological Sciences Online, a free online textbook on photobiology. ${ }^{3)}$ New chapters are added frequently.

The father of phototherapy is Niels Ryberg Finsen, 4) who first used sunlight, and then red light, to treat patients with smallpox in the 1800's. He received a Nobel Prize in 1903.
In recent times, a widely used phototherapy using visible light is for the treatment of neonatal jaundice, also known as hyperbilirubinemia. ${ }^{5)}$ Blue light isomerizes the bilirubin to make a water-soluble form, which can then be excreted in the bile, and to a lesser extent in urine. However, excessive light exposure, or the lack of cycled dark and light periods can produce unwanted side effects in the baby.

Another widely used phototherapy is for Seasonal Affective Disorder, or SAD. ${ }^{6}$ ) SAD is also known as winter depression. People who have normal mental health throughout most of the year, can experience depression during the winter. Most therapies for SAD use fluorescent light, but some use blue light. SAD appears to be a circadian rhythm problem.

The father of LLLT is Endre Mester, who in 1967 was using the newly invented red laser, and found that red light stimulated the growth of hair on shaved mice. ${ }^{7)}$

Since then, many wavelengths of visible and near infrared light from lasers, and now Light Emitting Diodes (LEDs), are being used for the treatment of various conditions, such as wound healing, and the relief of inflammation and pain.

The field of LLLT owes a huge debt of gratitude to Dr. Tina Karu for bringing the field of LLLT out of the dark ages, and into modern science. Her work on the action spectra for the stimulation of growth, and of DNA and RNA synthesis, and her correlation of the results with cytochrome $\mathrm{C}$ oxidase in the mitochondria was a quantum leap in the advancement of our understanding of one of the mechanisms, and probably the major mechanism, for the effectiveness of LLLT. ${ }^{8}$ )

An action spectrum is a plot of the relative effectiveness of different wavelengths of light in causing a particular biological response. Under ideal conditions it should mimic the absorption spectrum of the molecule that is absorbing the light, and whose photochemical alteration causes the biological effect. Thus, an action spectrum not only identifies the wavelength(s) that will have the maximum effect with the least dose of radiation, it also helps to identify the target of the radiation.

For example, the action spectrum for killing bacteria mimics the absorption spectrum of DNA. This result is understandable in view of the unique importance of DNA to a cell. This result tells us that if you want to inactivate DNA with the greatest efficiency, i.e., with 
the least dose of radiation, you should use the wavelength of ultraviolet (UV) radiation at the peak of the absorption and action spectra.

\section{Think of the different wavelengths of light as different drugs.}

Therefore, it is important to determine which drug is best, and also the optimum dose.

Based upon Dr. Karu's action spectrum, ${ }^{8)}$ several wavelengths are suggested to be optimal for LLLT, i.e., those around 400, 620, 680, 760, and $820 \mathrm{~nm}$. Most of these peaks are identifiable with cytochrome C oxidase, which resides in the mitochondria.

In eukaryotic cells, cytochrome C oxidase is the terminal enzyme of the respiratory chain, which mediates the transfer of electrons from cytochrome $\mathrm{C}$ to molecular oxygen. Ferrocytochrome $\mathrm{C}$ is oxidized, oxygen is reduced, and protons are pumped from the mitochondrial matrix to the cytosol. Free energy resulting from this redox chemistry is converted into an electrochemical potential across the inner membrane of the mitochondrion, which ultimately drives the production of ATP. Therefore, cytochrome $\mathrm{C}$ oxidase plays a central role in the bioenergetics of the cell.

However, that there are a lot of valleys in Dr. Karu's action spectrum, ${ }^{8}$ ) where the wavelengths are not very active per unit dose. Using a wavelength in one of the valleys might produce the effect that you want, but the dose necessary to produce the effect might be so high that unwanted side effects would probably also be produced.

Little is known about the targets for the stimulation effects produced by blue light at $400 \mathrm{~nm}$ in the action spectrum by Dr. Karu. However, flavoproteins like NADH-dehydrogenase could work as photoreceptors. The blue light region is an important area for future research, however, it does not penetrate deeply into tissues.

It must be remembered that most action spectra have been performed on a thin layer of cells. It is possible that the optimum wavelength under these conditions might not be the optimum wavelength for deep tissues. A longer wavelength that penetrates more deeply into tissues might produce the best effect in deep tissues even though that wavelength was not the most efficient in a thin layer of cells.
Another concept to be considered for LLLT is that two different wavelengths might produce a better effect under certain conditions such as wound healing. 9) For example, mast cells, neutrophils, and macrophages are the first cells to respond to a wound, and these cells respond best to $830 \mathrm{~nm}$ light. In contrast, fibroblasts, which are involved later, respond better to $633 \mathrm{~nm}$ light. Calderhead 9) suggests that it might be better to irradiate a wound first with $830 \mathrm{~nm}$ light, followed by $633 \mathrm{~nm}$ light, and then again with $833 \mathrm{~nm}$ light to activate the myofibroblasts.

The multiplicity of cell types in tissues, and the concept that they may respond better to different wavelengths, does complicate the phototherapy of tissues. It is just one more thing to keep in mind when planning experiments or clinical trials.

Mitochondria have a very complicated structure with multiple membranes, ${ }^{10)}$ and appear to be a major target for phototherapy. Mitochondria are thought to have evolved in very early bacteria. They contain their own DNA, which codes for 37 genes, 13 for subunits of respiratory complexes, 2 for ribosomal RNA, and 22 for the transfer RNAs necessary for protein synthesis.

There are a number of diseases and disorders correlated with a dysfunction of mitochondria, whose common theme is oxidative stress. Mitochondria also play a central role in many other metabolic tasks, such as: regulation of membrane potential, apoptosis-programmed cell death, cellular proliferation, regulation of cellular metabolism, certain heme synthesis reactions, steroid synthesis, and calcium signaling. It is little wonder, therefore, that mitochondria are a key target for phototherapy.

Mitochondria are the chief source of ATP, and are therefore considered to be the energy powerhouse for cells; ADP is recycled back into ATP, sugars and long chain fatty acids are broken down, steroids and lipids are synthesized, and DNA is replicated and transcribed.

Mitochondria are also an integral part of multiple cell signaling cascades. Proteins such as GTPases, kinases and phosphatases are involved in bi-directional communication between the mitochondrial reticulum, and the rest of the cell. ${ }^{11)}$

A recent paper entitled "The Double Life of ATP", 12) summarizes the fact that ATP is not just for energy, but is also a major factor in cell signaling, which allows 
cells and tissues throughout the body to communicate with one another.

ATP can become a signal when a firing neuron releases it from vesicles, along with neurotransmitter molecules. Many non-neuronal cells also release ATP using similar mechanisms. Enzymes then start breaking down ATP, sequentially removing phosphates to produce ADP, AMP, and adenosine. ATP and its breakdown products convey messages by binding to specific receptors on cells. ${ }^{12)}$

Figure 1 shows an overview of many signal transduction pathways. Cell signaling is an important response to LLLT.

Since DNA is the same in every cell of an organism, how is it possible for different organs to develop with different functions? Now we know that there are molecules that turn on or off the different genes in DNA in the different organs. Add to this the fact that once a gene is turned off or on, there are extensive signaling pathways to convey this information outward.

The so-called "bystander effect" was discovered using ionizing radiations. The observation was that radiation effects can be found in cells that are nearby, but not directly irradiated. ${ }^{14)}$ The first explanation that comes to mind is radiation scattering, however, there is now strong evidence for cell signaling as the mechanism. Also, there is evidence for the involvement of mitochondria in the signaling process. The bystander effect also occurs after exposure to light. ${ }^{15)}$

Calcium ions are intracellular messengers in many signal-transducing systems.

The intracellular level of calcium ions must be kept low because phosphate esters are prevalent, and calcium phosphates are very insoluble. The cytosolic level of calcium ions in unexcited cells is several orders of magnitude less than the extracellular concentration. Thus, the cytosolic calcium ion concentration can be abruptly raised for signaling purposes by transiently opening calcium channels in the plasma membrane or in an intracellular membrane.

The absorption of radiation in the infrared region, i.e., greater than $750 \mathrm{~nm}$, has been found to be very effective in a number of therapies using LLLT. However, the absorption of radiation in the infrared region results in

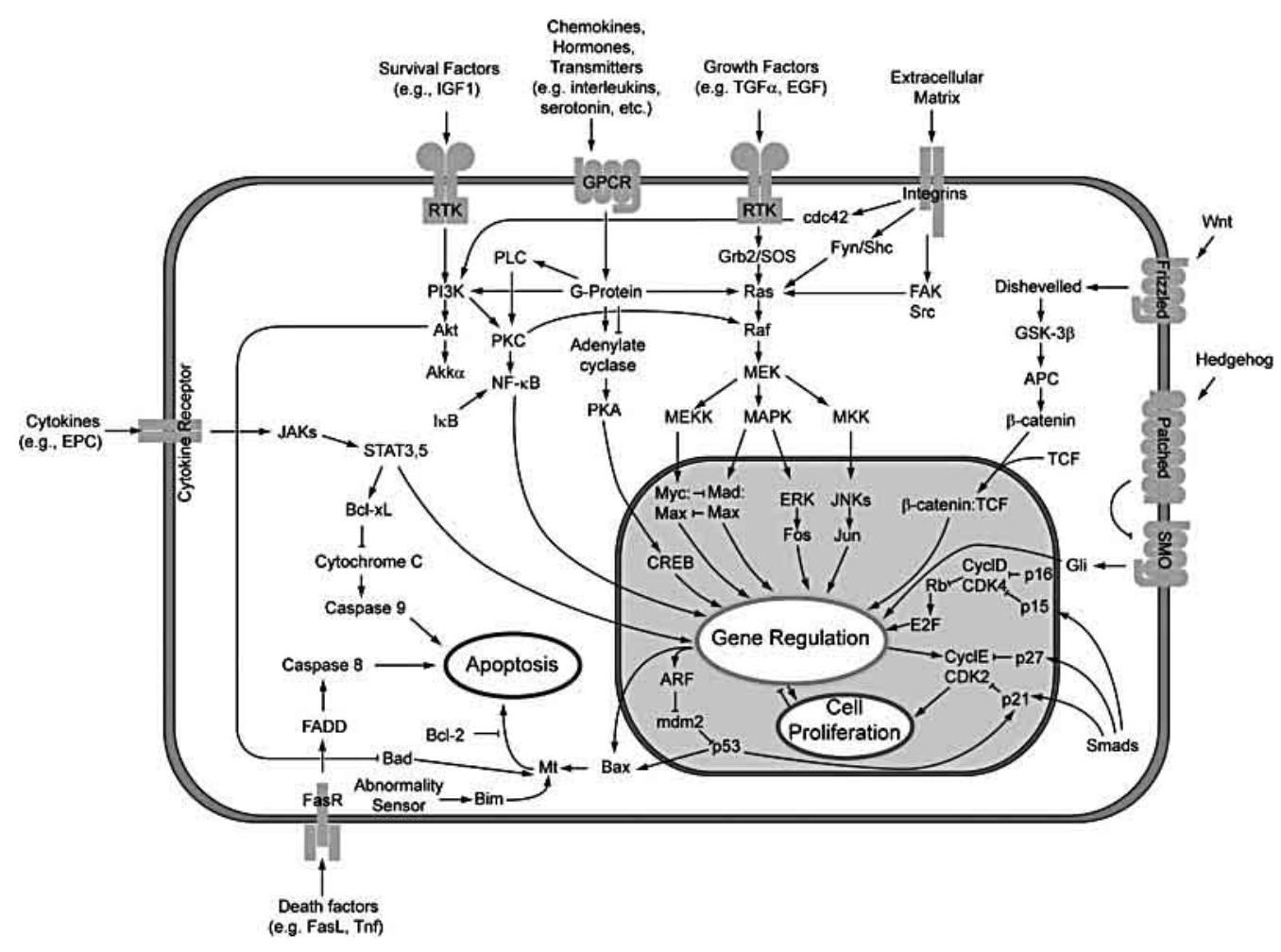

Figure 1: Overview of signal transduction pathways ${ }^{13)}$ 
molecular rotations and molecular vibrations, and is not expected to cause chemical changes in molecules.

There are a number of reports of the biological effects for LLLT at wavelengths both in the visible, and in the infrared region. For example, it was observed that irradiating fibroblasts in culture either at $633 \mathrm{~nm}$ or at 904 $\mathrm{nm}$ stimulated the synthesis of collagen. ${ }^{16)}$ What could be the mechanism for these similar results with such different wavelengths?

Some years ago I postulated that the effect of infrared radiation is to directly activate the calcium channels in membranes by molecular rotations and vibrations, which then results in the release of calcium, which activates the signal-transducing systems. ${ }^{17)}$

Dr. Karu's explanation for the photochemical effects of light on mitochondria (cytochrome $\mathrm{C}$ oxidase) involves a cascade of events that ultimately involves calcium signaling that effects growth (Figure 2).

My hypothesis is that infrared radiation skips the photochemical events on cytochrome $\mathrm{C}$ oxidase, and activates the calcium channels directly by its photophysical

\section{effects on membranes ${ }^{17)}$ (Figure 2).}

In support of this concept, Kujawa et al. ${ }^{19)}$ report that "Near-infrared laser light radiation $(810 \mathrm{~nm})$ induced long-term conformational transitions of red blood cell membrane, which were related to the changes in the structural states of both erythrocyte membrane proteins and lipid bilayer, and which manifested themselves as changes in fluorescent parameters of erythrocyte membranes, and lipid bilayer fluidity. This resulted in the modulation of membrane functional properties: changes in the activity of membrane ion pumps and, thus, changes in membrane ion flows."

Therefore, we need to be aware that light has both photochemical effects, and photophysical effects.

Over the years, a number of different enzymes have been observed to be activated by light, by a number of different mechanisms. The majority of the light activated enzymes that have been studied, however, are in plants and single cell organisms. e.g., 20)

The importance of the light activation of enzymes is that enzymes are catalysts. In principle, one photon can activate one enzyme molecule, which in turn can
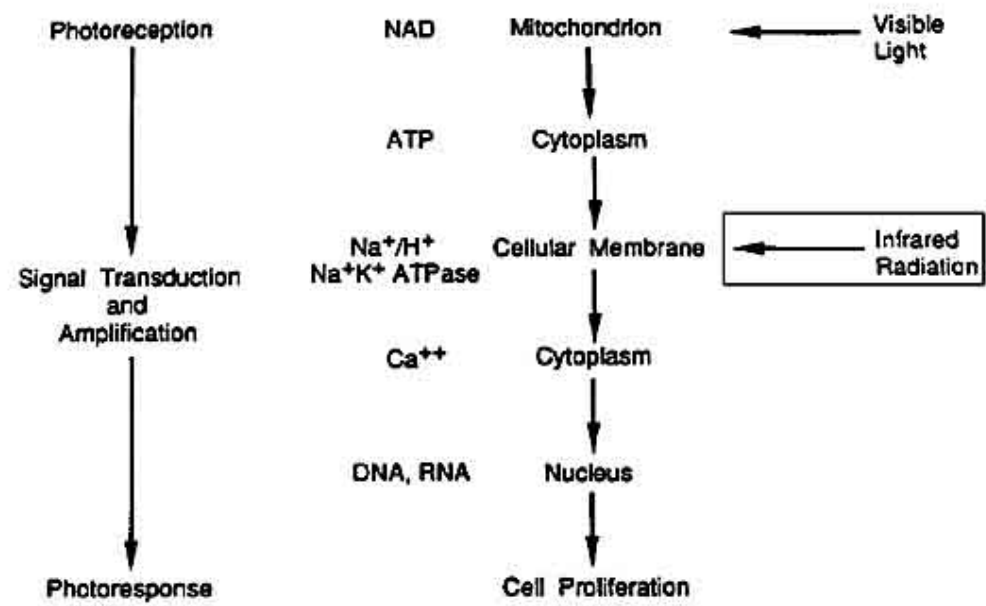

Figure 2: A model for the LLLT stimulation of biological systems [modified from Karu ${ }^{18)}$ ]. Biostimulation at $633 \mathrm{~nm}$ results from the absorption of light by components of the respiratory chain in the mitochondria (cytochrome $\mathrm{C}$ oxidase), which results in increased metabolism, which leads to signal transduction to other parts of the cell, and ultimately to the photoresponse. Because infrared radiation is expected to exert its photophysical effect on molecules that make up cellular membranes, I have added a boxed arrow to suggest that infrared radiation produces the same final biological result as does $633 \mathrm{~nm}$ radiation, but the two radiations start the cascade of metabolic events at different levels. ${ }^{17)}$ 
process many substrate molecules. This provides a huge amplification factor for initiating a biological response with light.

There are a number of ways, both direct and indirect, by which light can activate an enzyme.

1. Enzyme Induction. Light can induce the synthesis of enzymes. For example, laser irradiation at $633 \mathrm{~nm}$ has been shown to stimulate collagen synthesis in cutaneous wounds by enhancing the synthesis of Type I and Type II procollagen mRNA. ${ }^{21)}$

Current technology uses gene arrays ${ }^{22}$ ) for gene expression profiling. ${ }^{23)}$ Gene expression profiling provides information on all of the genes that are activated by a given treatment.

Using gene array analysis, Eells et al. ${ }^{24)}$ studied the induction of proteins in the retina after irradiation with $670 \mathrm{~nm}$ light. Although they did not provide a list of all of the gene products induced, they did provide a graph showing the different groups of proteins that were induced. More than $25 \%$ of the induced proteins were enzymes (Figure 3).

Table 1 summarizes a number of experiments with different cell types, listing the different molecules that are induced by LLLT.

The experiment that I would like to see performed is to irradiate cells at each of the wavelength peaks found by Dr. Karu in her action spectra experiments, i.e., 400, 620, 680, 760, and $820 \mathrm{~nm}$, and then perform gene array experiments at each wavelength. I would also like to see data for wavelengths further out in the infrared, such as 960 and $1060 \mathrm{~nm}$.

With lists of all of the genes induced, or not induced, by these different wavelengths, we would have a much better idea of what is going on during LLLT at each wavelength. It would also provide information relevant to the possible use of sequential treatments with several wavelengths. 9) I strongly recommend that such an experiment be run.

2. Activate an Enzyme-Substrate Complex. An example from UV photobiology is the photoreactivating enzyme, photolyase, which recognizes pyrimidine dimers in UV-damaged DNA, and forms an enzymesubstrate complex. In the presence of light in the range of $313-475 \mathrm{~nm}$, the enzyme uses the energy of the light to split the pyrimidine dimer back to monomeric pyrimidines, and the enzyme then goes hunting for another lesion, and then needs another light treatment when it finds a new lesion.

3. Activate an Enzyme Directly. This is generally done by stimulating a conformational change in the enzyme molecule itself. ${ }^{20)}$

4. Split Off an Inhibitor. A classical example of this is that the enzyme peroxidase is inhibited by the attachment of cyanide. Light at about $550 \mathrm{~nm}$ splits off the cyanide, and restores the activity of the enzyme.

Mayer and Heckel ${ }^{26)}$ wrote a review on "Biologically Active Molecules with a Light Switch". Small molecules, proteins, and nucleic acids have been modified to that they can be irreversibly activated with light (e.g., by

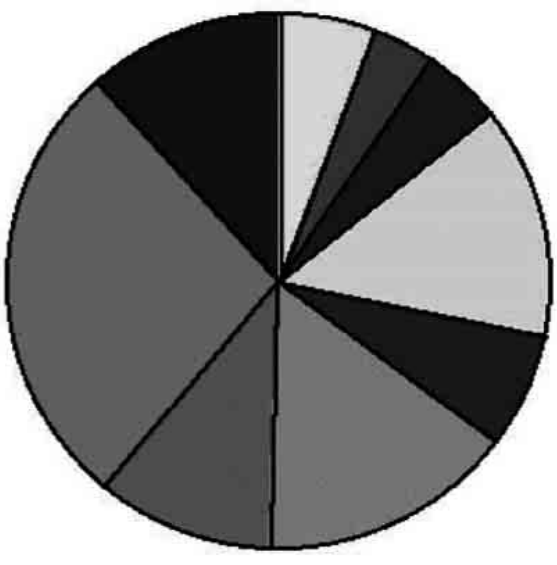

Figure 3: Gene expression profile for neural retina after $670 \mathrm{~nm}$ LED in methanol intoxicated rats. The figure shows the different groups of genes that were activated. Starting at 12 o'clock and going clockwise, the groups are: channels; cytochrome; peroxiredoxin; cell growth and maintenance; cell surface linked signal transduction; cell communication; signal transduction; enzymes (largest area); and transport. [modified from ${ }^{24}$ ] 
Table 1. Modulation of expression and secretion of molecules by LLLT. A summary of the literature [modified from ${ }^{25}$ )].

\begin{tabular}{|c|c|c|}
\hline Classification & Molecules & Biological effects of $\amalg T$ \\
\hline Growth factors & $\begin{array}{l}\text { BDNF, GDNF, bFGF, IGF-I, } \\
\text { KGF, PDGF, TGF- } \beta \text {, VEGF }\end{array}$ & $\begin{array}{l}\text { Proliferation, Differentiation, } \\
\text { Bone nodule formation }\end{array}$ \\
\hline Interleukins & IL-I $\alpha$, IL-6, IL-8, IL-2, IL-4 & Proliferation, Migration, Immunological activation \\
\hline Inflammatory cytokines & PGE2, COX-2, IL-I $\beta$, TNF- $\alpha$ & Inhibition of inflammation \\
\hline Small molecules & ATP, cGMP, ROS, $\mathrm{Ca}^{2+}, \mathrm{NO}$ & $\begin{array}{l}\text { Normalization of cell function, } \\
\text { Pain relief, Healing, Mediating cell activities, Migration, Angiogenesis }\end{array}$ \\
\hline
\end{tabular}

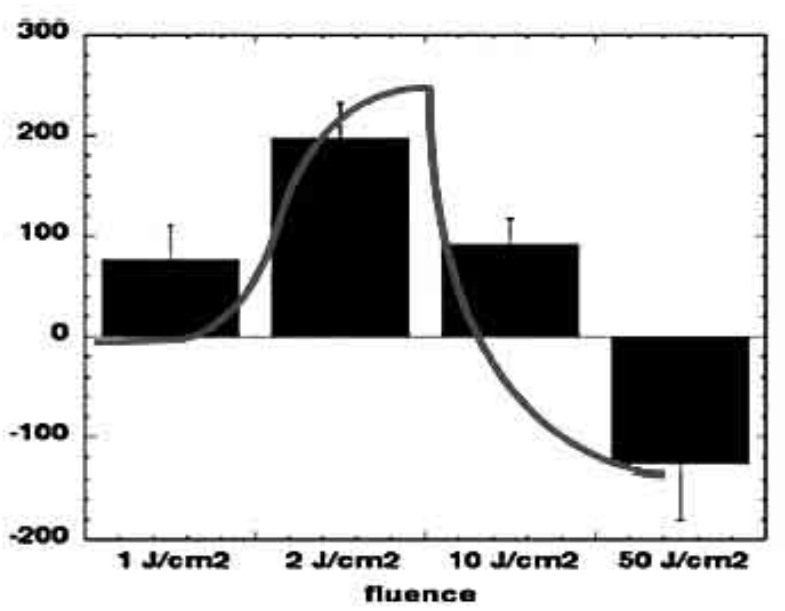

Figure 4: Biphasic dose response in measured difference in integrated area under the curve of the time course of wound size compared to the non-treated control. There is a clear benefit seen at $2 \mathrm{~J} / \mathrm{cm} 2$, but the high dose of $50 \mathrm{~J} / \mathrm{cm} 2$ provided a negative effect on the wound healing time curve [modified from ${ }^{27)}$.

splitting off an inhibitor), or they can be reversibly switched between an active and an inactive form with light, as is normally done with phytochrome in plants (see Photomorphogenesis, above).

\section{Enzyme Inactivation by Light.}

It is important to remember that enzymes can also be inactivated by light. For example, rye leaf catalase is inactivated by intense visible light. During photoinactivation, protein-bound heme is dissociated. Under physiological light conditions, however, this light inactivation is balanced by synthesis.
Huang et al. ${ }^{27}$ ) have published a review entitled "Biphasic Dose Response in Low Level Light Therapy". They emphasize that too much radiation is counter productive. Some doses of radiation are beneficial for wound healing, but too much radiation is damaging, as highlighted by the curve (Figure 4).

Again, think of the different wavelengths of light as different drugs.

Therefore, it is important to determine which drug is best, and also the optimum dose.

\section{Conclusion}

LLLT is very effective in stimulating wound healing and reducing inflammation and pain, and more and more of the molecular targets for the light in producing these effects are being discovered.

Probably the main target of visible light is cytochrome C oxidase, which resides in the mitochondria, which initiates a signal cascade that that initiates the cellular response. Other light activated molecules are enzymes. The targets for infrared radiation need further evaluation.

It would benefit the field immensely if gene array experiments were performed at the several wavelengths that have been shown to be the most effective in LLLT. Then we would have a catalog of genes that are activated by light. This would help with the design of proper protocols for different therapeutic strategies, and would provide valuable information for moving forward the field of LLLT into the main stream of science and medicine, where it belongs. 


\section{References}

1: Smith, KC (2010). Laser and LED Photobiology. Laser Therapy 19: 72-78.

2: Tuner, J and Hode, L (2007). The Laser Therapy Handbook, Prima Books AB

3: Photobiological Sciences Online (KC Smith, ed.), American Society for Photobiology, http://www.photobiology.info/

4: Finsen NR. Nobel Prize in Physiology or Medicine 1903. http://nobelprize.org/nobel_prizes/medicine/laureates/1903/finsen.html

5: Neonatal Jaundice, Wikipedia, http://en.wikipedia.org/wiki/Neonatal_jaundice

6: Sesonal Affective Disorder, Wikipedia, http://en.wikipedia.org/wiki/Seasonal_affective _disorder

7: Mester, E, Mester, AF, and Mester, A (1985). The Biomedical Effects of Laser Application. Lasers Surg. Med. 5: 31-39.

8: Karu, T (2008). Action Spectra: Their Importance for Low Level Light Therapy. Photobiological Sciences Online (KC Smith, ed.), American Society for Photobiology, http://www.photobiology.info/

9: Calderhead, RG (2007). The Photobiological Basics Behind Light-Emitting Diode (LED) Phototherapy. Laser Therapy 16: 97-108.

10: Mitochondria, Wikipedia, http://en.wikipedia.org/wiki/Mitochondrion

11: McBride, HM, Neuspiel, M and Wasiak, S (2006). Mitochondria: More Than Just a Powerhouse. Current Biol 16: R551-R560.

12: Khakh, BS and Burnstock, G (2009). The Double Life of ATP. Scientific American 301: 84-92.

13: Signal Transduction Pathways, Wikipedia, http://en.wikipedia.org/wiki/Signal_transduction

14: Sawant, SG, Randers-Pehrson, G, Metting, NF, and Hall, EJ (2001). Adaptive Response and the Bystander Effect Induced by Radiation in $\mathrm{C} 3 \mathrm{H}$ 10T1/2 Cells in Culture. Radiat Res 156: 177-180.

15: Tata, DB and Waynant, RW (2009). He-Ne laser enhanced cellular hydrogen peroxide production and induced modulations in metabolic activity in malignant human brain cancer: Evidence for a "bystander" effect. 2009 IEEE/NIH Life Science Systems and Applications Workshop (LiSSA 2009) http://ieeexplore.ieee.org/stamp/stamp.jsp?arnumber $=04906729$

16: Abergel RP, Meeker CA, Lam TS, Dwyer RM,
Lesavoy MA, Uitto J. (1984). Control Of Connective Tissue Metabolism By Lasers: Recent Developments And Future Prospects. J Am Acad Dermatol 11: 1142-50.

17: Smith, KC (1991). The Photobiological Basis of Low Level Laser Radiation Therapy. Laser Therapy 3: 19-24

18: Karu, T (1988). Molecular Mechanism of the Therapeutic Effet of Low-intensity Laser Radiation. Lasers in Life Science 2: 53-74.

19: Kujawa, J, Zavodnik, L, Zavodnik, I, Buko, V, Lapshyna, A, and Bryszewska, M. (2004). Effect of Low-Intensity (3.75-25 J/cm2) Near-Infrared (810 $\mathrm{nm}$ ) Laser Radiation on Red Blood Cell ATPase Activities and Membrane Structure. J Clinical Laser Med \& Surg 22: 111-117.

20: Hug, DH and Hunter, JK (1991) Photomodulation of Enzymes. J Photochem Photobiol B: Biol. 10: 222.

21: Saperia, D, Glassberg, E, Lyons, RF, Abergel, P, Baneux, P, Castel, JC, Dwyer, RM, and Uitto, J. (1986). Demonstration of elevated Type I and Type II procollagen mRNA levels in cutaneous wounds treated with helium-neon laser. Proposed mechanism for enhanced wound healing. Biochem Biophys Res Commun 138: 1123-28.

22: DNA Microarray, Wikipedia, http://en.wikipedia.org/wiki/DNA_microarray

23: Gene Expression Profiling, Wikipedia, http://en.wikipedia.org/wiki/Gene_expression _profiling

24: Eells, JT, Wong-Riley, MTT, VerHoeve, J, Henry, M, Buchman, EV, Kane, MP, Gould, LJ, Das, R, Jett, M, Hodgson, BD, Margolis, D, and Whelan, HT (2004). Mitochondrial Signal Transduction in Accelerated Wound and Retinal Healing by Nearinfrared Light Therapy. Mitochondrion 4: 559-567.

25: Gao, X and Xing, D (2009). Molecular Mechanisms of Cell Proliferation Induced by Low Power Laser Irradiation. J Biomed Sci 16: 4 (16 pages) [http://www.jbiomedsci.com/content/16/1/4].

26: Mayer, G and Heckel, A (2006). Biologically Active Molecules with a "Light Switch". Angew Chem Int Ed 45: 4900-4921.

27: Huang, Y-Y, Chen, A C.-H., Carroll, JD, Hamblin, MR (2009). Biphasic Dose Response in Low Level Light Therapy. Dose-Response 7: 353-383.

This paper is based upon a lecture presented at the ECl Conference "Light-Activated Tissue Regeneration and Therapy III", Burlington, VT (July 11-15, 2010). 